\title{
Joanna F. Haas
}

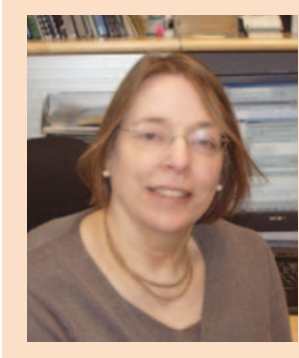

Joanna F. Haas, Vice-President, Pharmacovigilance, Genzyme Corporation

Joanna F. Haas joined Genzyme in 2000 after spending 15 years working in clinical development and pharmacovigilance for leading pharmaceutical companies in France, Germany, Austria and the US. She is a recognized leader in the management of human safety risks and has participated in industry and government collaborations in the US and Europe to optimize pharmacovigilance regulations and standards.

Why did Genzyme set up a distinct safety department?

Every company has to have a mechanism for dealing with safety because it's required by regulatory authorities, but at Genzyme we defined the responsibilities of the pharmacovigilance department in a much broader and more comprehensive fashion. In our conception, pharmacovigilance essentially has no borders, either geographically or in terms of the product life-cycle - it starts with preclinical safety information and goes right through to the last packet of pills sold on the market. And it's a global concept - all of the information has to end up in the same place and be dealt with in a uniform way to enable a single safety assessment from various types and sources of information.

\section{How unique is your approach?}

I don't know if it is unique but the comprehensive approach is definitely exceptional. In many companies the role of pharmacovigilance has been narrow and focused on post-marketing surveillance. Other safety activities are parsed to different parts of the organization. Such structures hinder the ability to recognize and respond to safety problems. This wall between research and commercialization has evolved over decades and attitudes are often difficult to change. For newer companies designing their systems from scratch our approach is a logical way to go.

What are the implications of separating safety from efficacy when establishing risk/benefit? You always have to look at both of them together. Although we have separate responsibility we're not isolating safety, because you can't view risk outside of the context of benefit; acceptable risk is different for psoriasis compared with pancreatic cancer. The pharmacovigilance representative works as an integral part of the clinical team in the course of the clinical development programme, but our voice is separate and distinct, which is important because others are focusing on efficacy, moving a project along and keeping it in budget. If there is a potential issue we might push towards early clarification or point to the probable need for a risk-management plan within the clinical development or post-marketing phase. The final decision on safety still falls to senior members of the organization, but concerns can be articulated more clearly with a single voice from pharmacovigilance.

How easy would it be for large multinationals to set up a similar model?

I spent many years working at other pharmaceutical companies and the organizational structures there are well entrenched, but if you begin working this way on a particular product or start off with such a group then I think it's easier. In addition to the core expertise needed for pharmacovigilance (medical expertise and an understanding of drug development and regulatory requirements) we also need people who can deal with basic science questions and can deal with pharmaco-epidemiological data once the product is on the market. It's a range of expertise that is not part of the traditional pharmacovigilance training and you won't get it all in one person.

\section{It is difficult to bring in this expertise?}

You need people who can assess information, including information to do with translational medicine and new scientific developments. We need to take advantage of cutting-edge science while realizing that development programmes need to incorporate validated methods.
For products that will be on the market you need to use methods that are available and will be accepted in medical practice.

\section{Does safety information flow voluntarily from different groups?}

We had to set up procedures to ensure it happens. Classically, during clinical development the pharmacovigilance group dealt primarily with serious adverse events, but developments in information technology mean that we can create methods for looking at non-serious adverse events, such as nonserious cases of liver disease, or tracking specific laboratory parameters that might be a marker for a specific problem. We can now take an issue of concern noted, for example, in preclinical studies and track it through clinical development across different indications. Common case definitions can be established, and common coding and data-management conventions can be used, across studies, which makes it easier to track safety prospectively.

Post-Vioxx, with the need for adequate riskmanagement plans, does your model have advantages over non-centralized models? Absolutely. Many of the procedures for dealing with clinical safety problems developed from the 'thalidomide paradigm': a readily recognizable problem that can be managed by bringing together case reports of serious unexpected events. But problems associated with Vioxx concern modest changes in the frequency of background events. The issue of increased suicidal behaviour in adolescents receiving selective serotonin reuptake inhibitors is even more subtle. Here the adverse reaction is indistinguishable from the natural history of the treated condition. So assessing risk here is a numbers game, both in clinical development and for marketed products. The first step is identifying potential issues. The second is setting up appropriate risk-management programmes that look at both clinical trials but also use registries, 'large simple safety studies' and pharmaco-epidemiology to assess and manage risks. If anything, Vioxx has reinforced the sense that we were right to place value on pharmacovigilance. I don't know whether some of our plans have been accelerated, but the value of them is all the more clear. 